\title{
Extracting Information about Chemical Bonding from Molecular Electron Densities via Single Exponential Decay Detector (SEDD)
}

\author{
Piotr de Silva ${ }^{\S \star a b}$, Jacek Korchowiec ${ }^{b}$, Nirmal Ram J. S. ${ }^{a}$, and Tomasz A. Wesolowski ${ }^{a^{\star}}$ \\ §SCS-Metrohm Foundation Award for best oral presentation
}

Abstract: The recently introduced molecular descriptor (Single Exponential Decay Detector - SEDD) [P. de Silva, J. Korchowiec, T. A. Wesolowski, ChemPhysChem 2012, 13, 3462] is used to visualize bonding patterns in molecules. In each point of space SEDD is simply related to the electron density:

$$
S E D D(\mathbf{r})=\ln \left[\frac{1}{\rho^{2}}\left(\nabla\left(\frac{\nabla \rho}{\rho}\right)^{2}\right)^{2}\right] \text {. }
$$

Either experimental or computed densities $\rho(\mathbf{r})$ can be used to evaluate SEDD. Here, maps of SEDD are obtained from theoretical densities and reveal such features as core electrons, chemical bonds, lone pairs and delocalization in aromatic systems. It is shown that SEDD provides fingerprints of aromaticity, which can be separated into geometric and electronic effects.

Keywords: Aromaticity · Bonding · Conceptual DFT · Electron density · Electron localization

\section{Introduction}

Electron localization is a fundamental concept in chemistry. It is directly related to such common elements of a chemist's language like bonds, lone pairs, shells, core and valence electrons. Despite their undoubted usefulness, these terms cannot be defined rigorously, which is a consequence of the quantum nature of electrons. Nevertheless, quantum chemistry has developed a multitude of methods which enables the capture of the local character of the electronic structure, including localized molecular orbitals ${ }^{[1-8]}$ and various molecular fields. ${ }^{[9-14]}$ Typically, these methods are based on the analysis of an approximate wave function, usually in the form of a single Slater determinant. On the other hand,

\footnotetext{
${ }^{\star}$ Correspondence: P. de Silva ${ }^{\text {ab }}$

Prof. T. A. Wesolowskia

E-mail: desilva@chemia.uj.edu.pl;

Tomasz.Wesolowski@unige.ch

aDepartment of Physical Chemistry

University of Geneva

30, quai Ernest-Ansermet

$\mathrm{CH}-1211$ Geneva 4

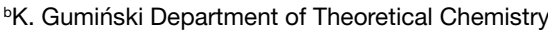

Faculty of Chemistry, Jagiellonian University

R. Ingardena 3, 30-060 Krakow, Poland
}

according to the first Hohenberg-Kohn theorem, the electron density itself contains all the information about any many-electron system. ${ }^{[15]}$ Unlike the wave function, the electron density is experimentally observable. Methods for elucidating information about electron localization only from the electron density are not so abundant in literature. Certainly the most prominent representative is Bader's charge density analysis. ${ }^{[16]}$ There have also been attempts to approximate the Electron Localization Function (ELF) or other scalar descriptors with functions that depend solely on the density. ${ }^{[17-20]}$

Recently, we have introduced the Single Exponential Decay Detector (SEDD), ${ }^{[21]}$ a new descriptor of the electronic structure, which is based only on the electron density. The analysis proved that SEDD reveals all the expected elements of the electronic structure in a similar fashion to ELF. In some cases, the bonding pattern revealed by SEDD shows an even better agreement with the character of interaction, e.g. for non-covalent bonding. The major advantage of SEDD is that being a semi-local density functional it is well-defined at any level of theory as well being directly applicable to experimental electron densities.

In the original paper, ${ }^{[21]}$ the electron densities used were calculated at B3LYP level of theory using QZ4P Slater Type
Orbitals (STO) basis set. The question of robustness of SEDD with respect to the basis set and the meaning of its values will be addressed in detail elsewhere. ${ }^{[22]}$ Here, we only mention that when Gaussian Type Orbitals (GTO) are employed, the qualitative picture is very similar. The discrepancies tend to diminish with the increase of the GTO basis set quality. The aim of the present study is to i) illustrate this conclusion by using electron densities obtained with GTOs and ii) examine how SEDD describes electron delocalization and aromatic character of molecules.

\section{Computational Details}

The geometries of all the systems were optimized at B3LYP/cc-pVQZ level using the Gaussian09 package. ${ }^{[23]}$ SEDD was calculated using a modified version of DGrid ${ }^{[24]}$ program and Paraview ${ }^{[25]}$ was used for visualization. SEDD color maps for all the systems were scaled between 2 and 15 from red to blue and the isovalue for 3D contours was fixed at 5. This particular choice, although arbitrary, is optimal for visualization purposes. It enables to distinguish between different elements of the bonding pattern and is universal in the sense that it applies to all systems examined so far by us. 


\section{Results}

To summarize and visualize the dependence of SEDD maps on the basis set used in quantum chemical calculations we have chosen three simple systems that already appeared in the original work, ${ }^{[21]}$ namely $\mathrm{LiH}, \mathrm{N}_{2}$ and CO. Fig. 1 shows SEDD color maps for these systems calculated from densities obtained with STO/QZ4P and GTO/cc-pVQZ basis sets. Lithium hydride (Fig. 1a) is an ionic compound bound by electrostatic interactions. No direct bonding between atoms, therefore, is visible. The maps for both basis sets reveal two disjoint regions that can be assigned to $\mathrm{Li}^{+}$ and $\mathrm{H}^{-}$ions. In the case of cc-pVQZ basis set the envelope of the molecule is less regular, which is the effect of poor description of the asymptotic behavior of density when GTOs are used. Similarly, the wrong description of cusp conditions with GTOs is the reason for higher values of SEDD at nuclei. These predictable shortcomings do not obscure the information about interactions in the $\mathrm{LiH}$ molecule, which is conveyed in SEDD maps, as STOs and GTOs provide the same qualitative description. For the $\mathrm{N}_{2}$ molecule (Fig. 1b) the same qualitative features are present irrespective of the basis set. In particular, electron cores, the direct $\mathrm{N}-\mathrm{N}$ bond and lone electron pairs can be discerned. The main difference concerns the description of the lone pairs, which are split in case of cc-pVQZ and compact for QZ4P basis set. The envelope of the molecule is less regular for GTOs as in the LiH example. The same observations apply to carbon monoxide (Fig. 1c), that is the picture obtained with GTOs is less regular but conveys the same information about bonding pattern as when STOs are used.

The main result of this work is the application of the SEDD analysis to describe delocalization in molecules due to their aromatic character. Many attempts to describe electron delocalization through the analysis of various molecular fields can be found in the literature.[26,27] Since SEDD is a new development, it is of interest to check how it performs for such systems. In particular, it is important whether it can discriminate between aromatic and non-aromatic systems just by using their total electron densities. We have selected a number of molecular systems containing a carbon-membered flat ring. They are either aromatic (benzene, $\mathrm{C}_{5} \mathrm{H}_{5}^{-}$), anti-aromatic $\left(\mathrm{C}_{5} \mathrm{H}_{5}{ }^{+}\right.$, cyclobutadiene) or non-aromatic (1,4-benzoquinone). In each case, SEDD is plotted in a form of $2 \mathrm{D}$ color maps or 3D isosurfaces.

The $\mathrm{C}_{5} \mathrm{H}_{5}^{-}$molecule is aromatic whereas $\mathrm{C}_{5} \mathrm{H}_{5}^{+}$is anti-aromatic. Their equilibrium structures have $\mathrm{D}_{5 \mathrm{~h}}$ and $\mathrm{C}_{2 \mathrm{v}}$ symmetries, respectively. Figs $2 \mathrm{a}$ and $2 \mathrm{~b}$
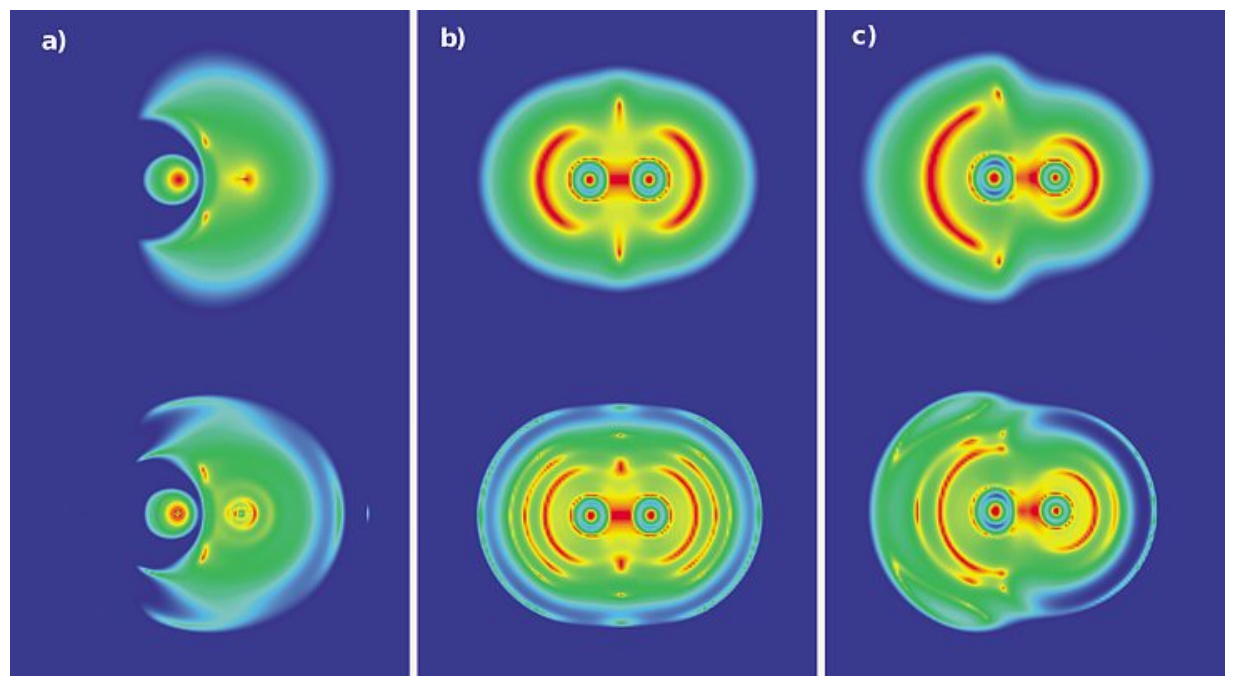

Fig. 1. Color plots of SEDD for $\mathrm{LiH}(\mathrm{a}), \mathrm{N}_{2}$ (b) and $\mathrm{CO}$ (c). Upper panel: STO/QZ4P basis set. Lower panel: cc-pVQZ basis set.

show SEDD maps in the planes of corresponding rings. Low values of SEDD (red color) reveal approximately exponentially decaying density, which is associated with increased electron localization. In both cases the $\mathrm{C}-\mathrm{C}$ and $\mathrm{C}-\mathrm{H}$ bonds are visible as well as the innermost electron shells in carbon atoms. For $\mathrm{C}_{5} \mathrm{H}_{5}^{-}$there is a continuous ring inside the molecule, while for $\mathrm{C}_{5} \mathrm{H}_{5}{ }^{+}$the ring seems to be disintegrated into smaller basins. We stress here, that by the continuous ring we mean only that it appears continuous at the color scale we have arbitrarily chosen. Additionally, other regions of low SEDD values are present in the vicinity of hydrogen atoms and outside the molecule, close to $\mathrm{C}-\mathrm{C}$ bonds. These finding are in line with what was reported in ref. [21], where SEDD was plotted for the same systems, but calculated in a STO basis set. Figs $3 a$ and $3 b$ show SEDD isosurfaces for the isovalue equal to 5.0. The qualitative difference between these two systems is readily apparent. For the aromatic $\mathrm{C}_{5} \mathrm{H}_{5}^{-}$a large delocalized structure encompassing the whole ring is present, while for $\mathrm{C}_{5} \mathrm{H}_{5}{ }^{+}$the SEDD isosurface is not that complex and reveals only a few disjoint basins outside the molecule. This example suggests that SEDD can reveal two fingerprints of aromaticity, namely the presence of a continuous ring in the middle of the carbon ring and a delocalized structure encapsulating the whole aromatic system. $\mathrm{C}_{5} \mathrm{H}_{5}^{-1+}$ systems differ mostly by two factors, i.e. number of electrons and the equilibrium geometry. To grasp the influence of these factors on SEDD profiles SEDD maps were plotted for the two systems with interchanged geometries, that is $\mathrm{C}_{5} \mathrm{H}_{5}^{-}$in
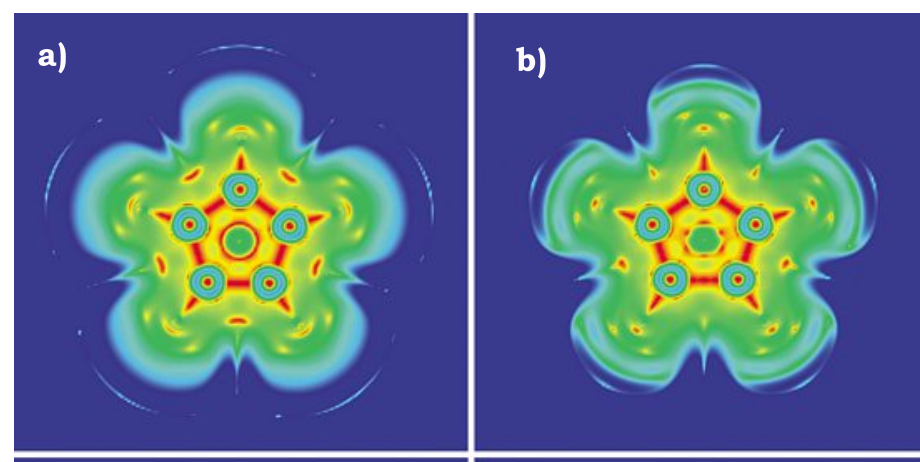

Fig. 2. Color plots of SEDD for $\mathrm{C}_{5} \mathrm{H}_{5}^{-}$(a), $\mathrm{C}_{5} \mathrm{H}_{5}{ }^{+}(\mathrm{b}), \mathrm{C}_{5} \mathrm{H}_{5}^{-}$in $\mathrm{C}_{5} \mathrm{H}_{5}^{+}$geometry (c) and $\mathrm{C}_{5} \mathrm{H}_{5}+$ in $\mathrm{C}_{5} \mathrm{H}_{5}$ geometry (d).
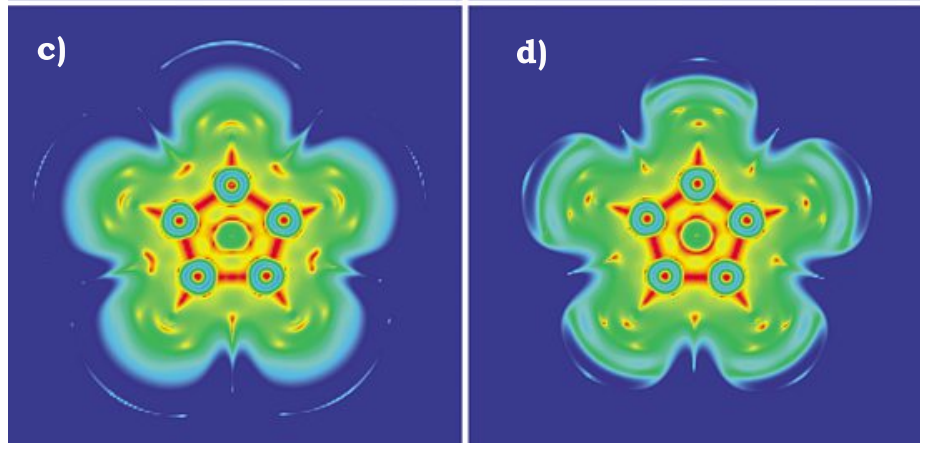
the geometry of $\mathrm{C}_{5} \mathrm{H}_{5}{ }^{+}$and vice versa. The results are collected in Figs $2 \mathrm{c}, 2 \mathrm{~d}, 3 \mathrm{c}$ and $3 d$. From these figures it can be concluded that the presence of the continuous SEDD ring is a geometric effect not directly related to aromaticity. This statement is supported by the fact that the ring is broken for $\mathrm{C}_{5} \mathrm{H}_{5}{ }^{-}$in $\mathrm{C}_{5} \mathrm{H}_{5}{ }^{+}$geometry (Fig. 2c) and is continuous for $\mathrm{C}_{5} \mathrm{H}_{5}{ }^{+}$in $\mathrm{C}_{5} \mathrm{H}_{5}^{-}$geometry (Fig. 2d), which is clearly not aromatic. On the other hand, SEDD contours in Figs 3c and $3 \mathrm{~d}$ strongly indicate that the presence of delocalized SEDD structures can be a signature of aromaticity. This delocalization appears for $\mathrm{C}_{5} \mathrm{H}_{5}^{-}$in $\mathrm{C}_{5} \mathrm{H}_{5}^{+}$geometry (Fig. 3c), while it is not present for $\mathrm{C}_{5} \mathrm{H}_{5}^{+}$ in $\mathrm{C}_{5} \mathrm{H}_{5}^{-}$geometry (Fig. 3d). Due to the distortion from the equilibrium $\mathrm{D}_{5 \mathrm{~h}}$ geometry, the isosurface is disintegrated into three parts, however, it retains its characteristic features.

To confirm that delocalized domains are fingerprints of aromaticity, we compare three systems containing a flat carbonmembered ring, namely benzene, cyclobutadiene and 1,4-benzoquinone, which are aromatic, anti-aromatic and nonaromatic, respectively. The 3D contour maps (Fig. 4) of SEDD for these molecules confirm that the presence of a SEDD=5.0 isosurface that extends over the whole carbon ring is a fingerprint of aromaticity, at least for this class of systems. For benzene (Fig. 4a) this structure is composed of rings embracing $\mathrm{C}-\mathrm{C}$ bonds, which merge together to form a single localization domain. For the anti-aromatic cyclobutadiene (Fig. 4b) only small localization domains are visible, which are located in the vicinity of the formally double bonds, but do not form a ring around them. In case of the nonaromatic 1,4-benzoquinone (Fig. 4c) two rings around the double bonds are present. They are connected with a structure inside the carbon ring however there is no delocalization over other $\mathrm{C}-\mathrm{C}$ bonds.

\section{Conclusions}

The present work shows that SEDD is a meaningful descriptor of bonding patterns in molecular systems. It is robust with respect to the type of the basis set used, provided that it is sufficiently large. The comparison of the results between STO/ QZ4P and GTO/cc-pVQZ basis sets reveals that in both cases SEDD gives a coherent picture of bonding. A more detailed discussion of basis set effects is beyond the scope of this work and will be published elsewhere. ${ }^{[22]}$

Application of SEDD to delocalized systems shows that aromaticity can be detected just by analyzing the total electron density without referring to molecular orbitals. The analysis shows that some

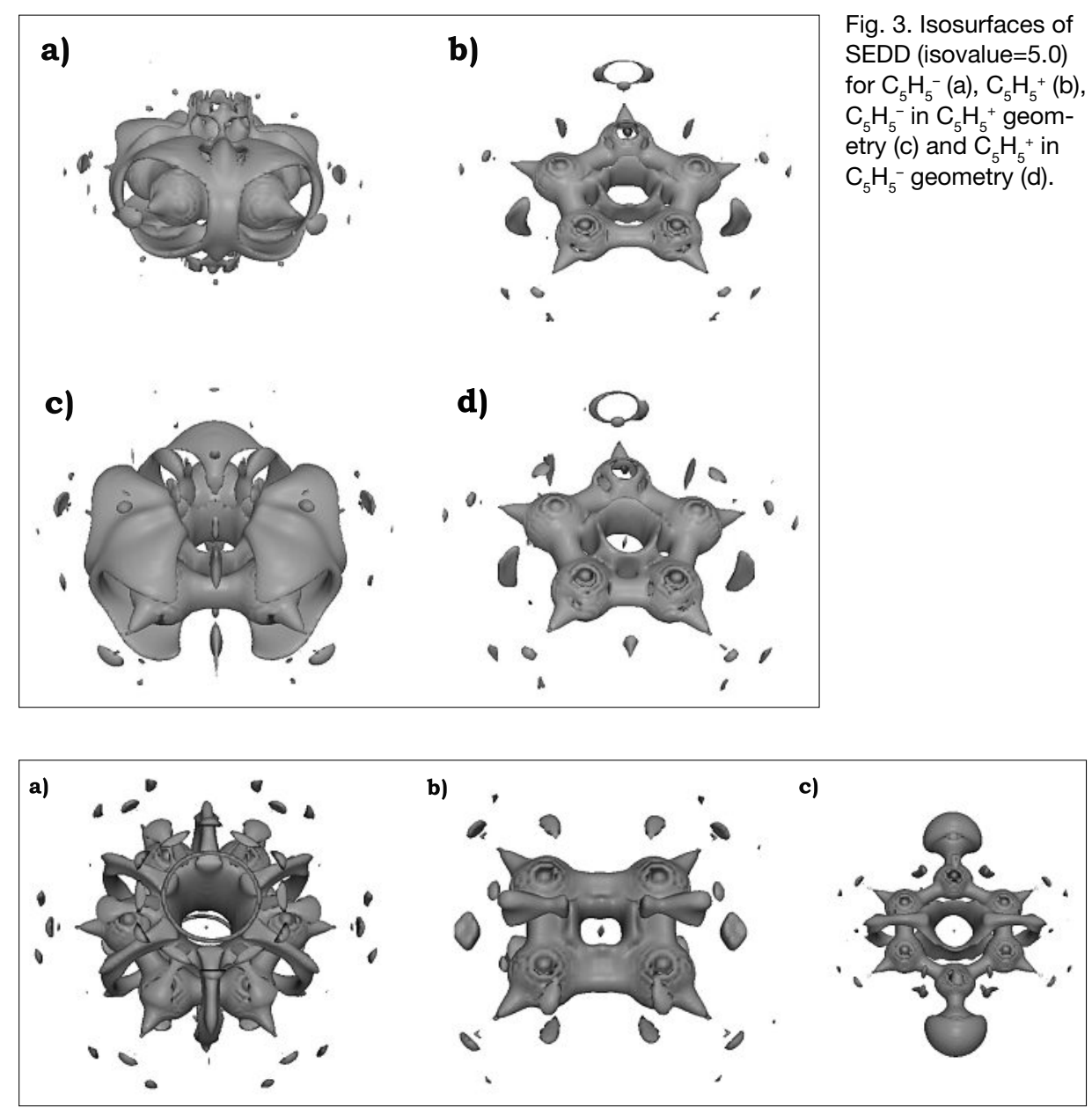

Fig. 4. Isosurfaces of SEDD (isovalue=5.0) for benzene (a), cyclobutadiene (b) and 1,4-benzoquinone (c).

features of SEDD in aromatic systems are a direct consequence of the geometry and are triggered by bond equalization in the aromatic ring. This applies to the emergence of the continuous central ring in SEDD maps. Another signature of aromaticity, which is revealed by SEDD, is the appearance of domains with low SEDD values that are delocalized over the whole aromatic ring. This feature is clearly an electronic effect, which persists when the geometry is distorted from the equilibrium structure.

Since SEDD depends only on the electron density, in principle, it can be determined experimentally. This opens a new way for a direct comparison of theoretical and experimental results in the field of bonding analysis. We believe that such applications will appear in the near future and will help to bridge the theory with the experiment.

\section{Acknowledgements}

This work was supported by the International $\mathrm{PhD}$-studies program at the Faculty of Chemistry Jagiellonian University within the Foundation for Polish Science MPD Program co-financed by the EU European Regional Development Fund and the Grant from Swiss National Science Foundation
(200020/134791/1). NR acknowledges the support by Federal Commission for Scholarships for Foreign Students (FCS) offered by Switzerland.

Received: February 22, 2013

[1] S. F. Boys, Rev. Mod. Phys. 1960, 32, 296.

[2] C. Edmiston, K. Ruedenberg, Rev. Mod. Phys. 1963, 35, 457

[3] J. Pipek, P. G. Mezey, J. Chem. Phys. 1989, 90, 4916.

[4] A. Reed, L. A. Curtiss, F. Weinhold, Chem. Rev. 1988, 88,899 .

[5] H. Stoll, G. Wagenblast, H. Preuss, Theor. Chim. Acta 1980, 57, 169.

[6] F. Aquilante, T. B. Pedersen, A. S. de Meras, H. Koch, J. Chem. Phys. 2006, 125, 174101.

[7] P. de Silva, M. Giebułtowski, J. Korchowiec, Phys. Chem. Chem. Phys. 2012, 14, 546.

[8] P. de Silva, M. Makowski, J. Korchowiec, Chimia 2012, 66, 178.

[9] A. D. Becke, K. E. Edgecomb, J. Chem. Phys. 1990, $92,5397$.

[10] B. Silvi, A. Savin, Nature 1994, 371, 683.

[11] A. Savin, O. Jepsen, J. Flad, O. K. Andersen, H. Preuss, H. G. von Schnering, Angew. Chem. 1992, 104, 186.

[12] H. L. Schmider, A. D. Becke, J. Mol. Struct.: THEOCHEM 2000, 527, 51.

[13] M. Kohout, Int. J. Quantum Chem. 2004, 97, 651.

[14] R. F. Nalewajski, P. de Silva, J. Mrozek, J. Mol. Struct.: THEOCHEM 2010, 954, 57.

[15] P. Hohenberg, W. Kohn, Phys. Rev. 1964, 136, B864. 
[16] R. Bader, 'Atoms in Molecules: A Quantum Theory', Oxford University Press, Oxford, 1994.

[17] V. G. Tsirelson, A. I. Stash, Chem. Phys. Lett. 2002, 351, 142.

[18] K. Finzel, Y. Grin, M. Kohout, Theor. Chem. Acc. 2012, 131, 1.

[19] V. G. Tsirelson, A. I. Stash, V. V. Karasiev, S. Liu, Comput. Theor. Chem. 2012, 1006, 92.

[20] S. R. Gadre, S. A. Kulkarni, R. K. Pathak, J. Chem. Phys. 1993, 98, 3574.

[21] P. de Silva, J. Korchowiec, T. A. Wesolowski, ChemPhysChem 2012, 13, 3462.

[22] N. Ram, P. de Silva, J. Korchowiec, T. A. Wesolowski, to be published.
[23] Gaussian 09, M. J. Frisch, G. W. Trucks, H. B. Schlegel, G. E. Scuseria, M. A. Robb, J. R. Cheeseman, G. Scalmani, V. Barone, B. Mennucci, G. A. Petersson, H. Nakatsuji, M. Caricato, X. Li, H. P. Hratchian, A. F. Izmaylov, J. Bloino, G. Zheng, J. L. Sonnenberg, M. Hada, M. Ehara, K. Toyota, R. Fukuda, J. Hasegawa, M. Ishida, T. Nakajima, Y. Honda, O. Kitao, H. Nakai, T. Vreven, J. A. Montgomery, Jr., J. E. Peralta, F. Ogliaro, M. Bearpark, J. J. Heyd, E. Brothers, K. N. Kudin, V. N. Staroverov, R. Kobayashi, J. Normand, K. Raghavachari, A. Rendell, J. C. Burant, S. S. Iyengar, J. Tomasi, M. Cossi, N. Rega, J. M. Millam, M. Klene, J. E. Knox, J. B. Cross, V. Bakken,
C. Adamo, J. Jaramillo, R. Gomperts, R. E. Stratmann, O. Yazyev, A. J. Austin, R. Cammi, C. Pomelli, J. W. Ochterski, R. L. Martin, K. Morokuma, V. G. Zakrzewski, G. A. Voth, P. Salvador, J. J. Dannenberg, S. Dapprich, A. D. Daniels, Ö. Farkas, J. B. Foresman, J. V. Ortiz, J. Cioslowski, and D. J. Fox, Gaussian, Inc., Wallingford CT, 2009.

[24] M. Kohout, DGrid, version 4.6, Radebeul, 2011

[25] A. Henderson, ParaView Guide, A Parallel Visualization Application, Kitware Inc., 2012.

[26] J. Poater, M. Duran, M. Solà, B. Silvi, Chem. Rev. 2005, 105, 3911.

[27] G. Merino, A. Vela, T. Heine, Chem. Rev. 2005, 105,3812 